9.. Alley SC, Harris JR, Cao A, et al. Tisotumab vedotin induces antitumor activity through MMAE-Mediated, Fc-Mediated, and Fab-Mediated Effector Functions In Vitro [abstract]. Cancer Res. 2019;79(13 Suppl):Abstract 221.

10. Breij $E$, de Goeij B, Verploegen $S$, et al. An antibody-drug conjugate that targets tissue factor exhibits potent therapeutic activity against a broad range of solid tumors. Cancer Res $2014 ; 74(4): 1214-1226$.

http://dx.doi.org/10.1136/jitc-2020-SITC2020.0617

\section{VEDOTIN ADCS INDUCE ER STRESS AND ELICIT HALLMARKS OF ICD ACROSS MULTIPLE CANCER INDICATIONS}

${ }^{1}$ Kerry Klussman*, ${ }^{1}$ Elena-Marie Tenn, ${ }^{1}$ Shaylin Higgins, ${ }^{1}$ Rebecca Mazahreh, ${ }^{1}$ Katie Snead, ${ }^{1} J o s e p h$ Hamilton, 'Bryan Grogan, 'Johann Sigurjonsson, ${ }^{2}$ Anthony Cao, 'Shyra Gardai, ${ }^{1}$ Bernard Liu. 'Seattle Genetics, Inc., Bothell, WA, USA; ${ }^{2}$ Celgene/Bristol-Myers Squibb, San Francisco, CA, USA

Background Effective cancer treatment requires durable elimination of malignant cells. Cytotoxic chemotherapeutic agents used to treat cancer often show initial anti-tumor efficacy, but fail to produce long-term durable responses in patients. The elicitation of durable responses and improved survival in response to cytotoxic agents may be associated with the induction of innate and adaptive immune response to the cancer. For example, tumor cells undergoing apoptosis following exposure to some cytotoxic agents emit immunostimulatory damage-associated molecular patterns (DAMPs), this form of cell death is termed immunogenic cell death (ICD). ICD can promote the recruitment and activation of both the innate and adaptive immune system, providing an additional mechanism to drive an anti-tumor response.

Methods Vedotin-based antibody drug conjugates (ADCs) drive cytotoxicity in tumor cells by engaging tumor antigens on the cell surface, internalizing with the cell surface antigen, and delivering monomethyl auristatin E (MMAE) payload. Following intracellular delivery, MMAE induces mitotic arrest, as well as an endoplasmic reticulum (ER) stress response resulting from microtubule disruption. Following tumor cell treatment, indicators of the ER stress response are observed with vedotin-based ADCs including induction of phospho-JNK and CHOP, This mechanism of MMAE induced ER stress results in emission of hallmark ICD DAMPs including cell-surface calreticulin, extracellular release of HMGB1 and ATP. In this presentation we highlight the ability of MMAE to induce the hallmarks of ICD in multiple cancers across different tissue origins using distinct valine-citrulline-MMAE (vedotin)-based ADCs.

Results The culmination of these ICD hallmarks resulted in innate immune cell activation in vitro and in vivo in mouse xenograft models. Tumor bearing mice treated with vedotinbased ADCs resulted in the promotion of immune cell recruitment and activation in tumors. Analysis of immune activation by vedotin-based ADCs included production of innate cytokines and upregulation of HLA/MHC-Class I/II expression, which supports a role in activating both the innate and adaptive immune response. To further our understanding of the potent and broad ability of vedotin ADCs to induce ICD, we have also begun to examine the ICD potential of different classes of ADC payloads including other microtubule inhibitors (auristatins and maytansines), and DNA damaging agents
(DNA alkylators or topoisomerase inhibitors). Initial data indicate differences in ICD induction by these agents.

Conclusions These results help build the rationale for vedotinbased ADCs as preferred partners for immune checkpoint blockade agents.

Ethics Approval Studies with human samples were performed according to institutional ethics standards. Animal studies were approved by and conducted in accordance with Seattle Genetics Institutional Care and Use Committee protocol \#SGE-029.

http://dx.doi.org/10.1136/jitc-2020-SITC2020.0618

\section{EVALUATING THE EFFECTIVENESS OF TARGETED ADC THERAPY IN A PATIENT-DERIVED EX VIVO TUMOROID MODEL, 3D-EX, FOR QUANTITATIVE TUMOR CELL KILLING}

Jenny Kreahling, Jared Ehrhart, Mibel Pabon*, Stephen Iwanowycz, Tina Pastoor, Soner Altiok. Nilogen Oncosystems, Tampa, Florida, USA

Background Antibody drug conjugates (ADCs) are an effective tool for site directed delivery of cytotoxic agents to cancer cells. Tailoring of ADC-specificity to the uniqueness of a patient's tumor can aid in direct-targeting of tumor cells and potentially improve drug responsiveness. Here we evaluate the potential of using an ADC therapy for targeted tumor cell death and immune cell activation in combination with checkpoint inhibitors in 3D tumoroids.

Methods All human tumor samples were obtained with proper patient consent and IRB approval. Fresh patient tumor tissue of various histologic types including CRC and NSCLC were processed to generate uniform sized live $3 \mathrm{D}$ tumoroids measuring $150 \mu \mathrm{m}$ in size. Treatment groups included a conjugated ADC therapeutic antibody alone or in combination with PD-1/ PD-L1 inhibitors. Culture supernatants were collected for multiplex analysis of cytokine release in media. Additionally, flow cytometry was used to assess the activation profile of resident immune cells in combination with high-content confocal imaging to determine extent of tumor cell death in the intact tumor extracellular matrix.

Results Using fresh patient-derived tumoroids, we observed ADC-mediated cell death and activation of immune cells within the tumor microenvironment. Production of pro-inflammatory cytokines correlated with increased activation of tumor infiltrating immune cell populations. The improved immune response led to increased tumor cell killing within the $3 \mathrm{D}$ tumor microenvironment observed by high-content confocal imaging.

Conclusions In this study we demonstrate that our physiologically relevant $3 \mathrm{D}$ tumoroid model is an effective system to assess novel antibody drug conjugates and to develop rational drug combinations with other immuno-oncology agents. Furthermore, implementation of 3D-EX platform, in the clinical setting, may also allow for determination of the most effective combinatorial immuno-oncology treatment strategies for individualized patient care.

Ethics Approval The study was approved by Chesapeake IRB Pro00014313.

http://dx.doi.org/10.1136/jitc-2020-SITC2020.0619 